\title{
Xanthogranulomatous Cholecystitis Masquerading as Gallbladder Cancer: Can It Be Diagnosed Preoperatively?
}

\author{
Ashwin Rammohan, Sathya D. Cherukuri, Jeswanth Sathyanesan, \\ Ravichandran Palaniappan, and Manoharan Govindan
}

The Institute of Surgical Gastroenterology \& Liver Transplantation, Centre for GI Bleed, Division of HPB Diseases, Stanley Medical College Hospital, Old Jail Road, Chennai 600 001, India

Correspondence should be addressed to Ashwin Rammohan; ashwinrammohan@gmail.com

Received 31 August 2014; Revised 3 October 2014; Accepted 4 October 2014; Published 27 October 2014

Academic Editor: Gianfranco D. Alpini

Copyright (C) 2014 Ashwin Rammohan et al. This is an open access article distributed under the Creative Commons Attribution License, which permits unrestricted use, distribution, and reproduction in any medium, provided the original work is properly cited.

\begin{abstract}
Background. Xanthogranulomatous cholecystitis (XGC) is often misdiagnosed as gallbladder cancer (GBC). We aimed to determine the preoperative characteristics that could potentially aid in an accurate diagnosis of XGC masquerading as GBC. Methods. An analysis of patients operated upon with a preoperative diagnosis of GBC between January 2008 and December 2012 was conducted to determine the clinical and radiological features which could assist in a preoperative diagnosis of XGC. Results. Out of 77 patients who underwent radical cholecystectomy, 16 were reported as XGC on final histopathology (Group A), while 60 were GBC (Group B). The incidences of abdominal pain, cholelithiasis, choledocholithiasis, and acute cholecystitis were significantly higher in Group A, while anorexia and weight loss were higher in Group B. On CT, diffuse gallbladder wall thickening, continuous mucosal line enhancement, and submucosal hypoattenuated nodules were significant findings in Group A. CT findings on retrospect revealed at least one of these findings in $68.7 \%$ of the cases. Conclusion. Differentiating XGC from GBC is difficult, and a definitive diagnosis still necessitates a histopathological examination. An accurate preoperative diagnosis requires an integrated review of clinical and characteristic radiological features, the presence of which may help avoid radical resection and avoidable morbidity in selected cases.
\end{abstract}

\section{Introduction}

Xanthogranulomatous cholecystitis (XGC) is an inflammatory disease of the gallbladder, characterised by a focal/diffuse destructive inflammatory process followed by marked proliferative fibrosis along with infiltration of macrophages and foamy cells [1]. Its incidence ranges from 0.7 to $10 \%$ [1-3]. XGC exhibits similar imaging and intraoperative findings as those of gallbladder cancer (GBC), leading to its frequent misdiagnosis [2-5]. Imaging does shed some light on this conundrum preoperatively, but diagnosis is often a postoperative histological surprise [6-12]. These lesions are easily confused with more sinister neoplasms, and hence there needs to be an increased awareness of this tumour mimic, particularly in endemic areas [6-12]. Identifying the preoperative differences between XGC and GBC is imperative, as it would help avert unnecessary morbidity especially in the form of radical surgery. This study aimed to determine the preoperative characteristics of XGC that could potentially aid in an accurate diagnosis of XGC masquerading as GBC.

\section{Methods}

An analysis from a prospectively collected database of patients operated-upon in our department with a preoperative diagnosis of GBC between January 2008 and December 2012 was done. All patients who underwent a radical cholecystectomy were considered for the study. Out of a total of 77 patients, 76 were included in the study; one patient had GBC associated with XGC and was excluded from the analysis. Based on their final histopathology, the patients were divided into two groups. Out of the 76 patients, 16 were reported as XGC on final histopathology (Group A), while 60 were GBC (Group B). Data was collected with regard to 


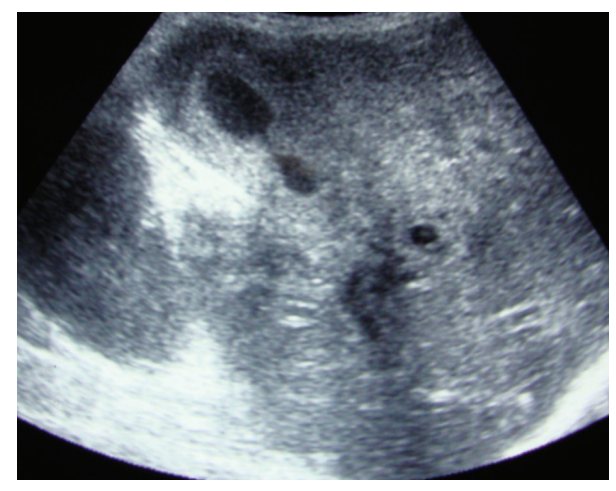

FIGURE 1: Ultrasound showing smooth uniform thickening of gallbladder wall.

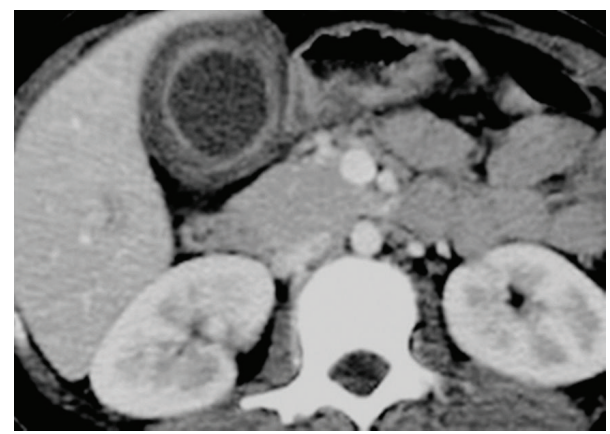

FIGURE 2: Contrast-enhanced CT showing smooth circumferential gallbladder wall thickening with a continuous contrast-enhanced mucosal line.

clinical features, tumor markers (CEA, CA19.9), and radiological investigations \{ultrasonogram (USG) and/or CT\}. Particular stress was given on certain radiological features like the thickness of the gallbladder wall, patterns of wall thickening (focal versus diffuse), continuity of mucosal line (continuous versus disrupted), enhancement characteristics of mucosa (homogeneous versus heterogeneous), presence of submucosal hypoattenuated nodules or bands, and presence or absence of enlarged lymph nodes (Figures 1 and 2). Clinical and radiological features were compared between $\mathrm{XGC}$ and GBC to determine features which could assist in a preoperative diagnosis of XGC.

\section{Statistical Analysis}

Nominal variables were comparedusing the chi-square or Fisher's exact probability test, while continuous variables were compared using Student's $t$-test. A $P$ value $<0.05$ was considered statistically significant. All statistical analyses were performed using SPSS 20.0 statistical package (SPSS, Chicago, IL, USA).

\section{Results}

Out of 77 patients with resectable disease who underwent radical cholecystectomy, 16 were reported as XGC (Group A), while 60 patients had a histopathology of GBC (Group B)

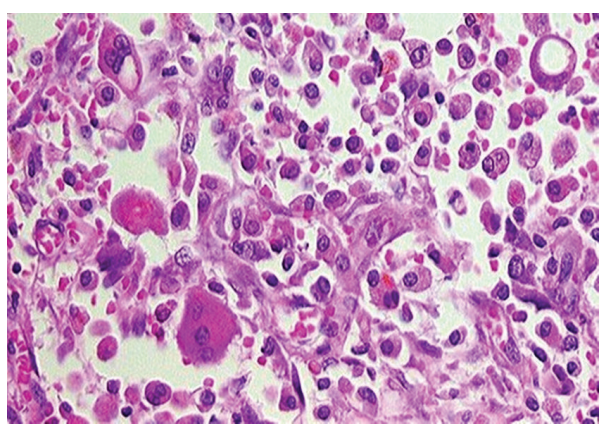

FIGURE 3: Histopathology slide of XGC showing foamy macrophages and giant cells in the wall of the gallbladder.

TABLE 1: Comparison of clinical features and tumour markers between XGC and GBC.

\begin{tabular}{lccc}
\hline & XGC & GBC & $P$ value \\
\hline Age (mean \pm SD) & $56.2 \pm 12.4$ & $58 \pm 11.1$ & NS \\
Male/female & $6 / 9$ & $25 / 35$ & NS \\
Abdominal pain (\%) & $16(100)$ & $37(61)$ & $P<0.01$ \\
Acute cholecystitis (\%) & $12(75)$ & $9(15)$ & $P<0.01$ \\
Cholelithiasis (\%) & $11(68.8)$ & $17(28.3)$ & $P<0.01$ \\
Choledocholithiasis $(\%)$ & $4(25)$ & $2(3.3)$ & $P<0.01$ \\
Loss of weight $(\%)$ & $3(18.8)$ & $36(60)$ & $P<0.01$ \\
Loss of appetite $(\%)$ & $9(56)$ & $42(70)$ & $P<0.05$ \\
Diabetes $(\%)$ & $5(31.3)$ & $18(30)$ & NS \\
Jaundice & $2(12.5)$ & $8(13.3)$ & NS \\
Palpable mass & $5(18.8)$ & $7(11.7)$ & NS \\
Tumour markers & & & \\
$\quad$ CEA $(\geq 4$ ng/mL) & 0 & 49 & $P<0.01$ \\
CA19.9 $(\geq 20 \mathrm{IU} / \mathrm{mL})$ & 2 & 41 & $P<0.01$ \\
\hline
\end{tabular}

(Figure 3). One patient had GBC associated with XGC and was excluded from the study. Analysis of the clinical features revealed a higher incidence of abdominal pain and acute cholecystitis in Group A. Cholelithiasis and choledocholithiasis were also more commonly seen in Group A. The incidences of anorexia and weight loss were significantly higher in Group B. There were no significant differences with respect to age, gender, presence of jaundice, and the presence of a palpable mass. Tumour markers (serum CEA and CA19.9) were found to be significantly elevated in patients with GBC (Table 1).

On comparison of radiological features, patients with XGC were more frequently found to have a diffuse gallbladder wall thickening, continuous mucosal line enhancement, and submucosal hypoattenuated nodules or bands (Figures 1 and 2 and Table 2). There were no significant differences in the thickness of gallbladder wall or enlargement of lymph nodes between the groups. On retrospect, at least one of these findings was noted in $68.7 \%$ (11/16) cases with XGC. 
TABLE 2: Comparison of radiological findings between XGC and GBC.

\begin{tabular}{lccc}
\hline Radiological findings & XGC & GBC & $P$ value \\
\hline GB wall thickness (mean \pm SD, mm) & $14.1 \pm 4.9$ & $13.6 \pm 6.1$ & NS \\
Diffuse GB wall thickening & $4(36.6 \%)$ & $4(6.6 \%)$ & \\
Continuous mucosal line & $8(50 \%)$ & $6(10 \%)$ & $P<0.01$ \\
Submucosal hypoattenuated nodules/band & $9(56.2 \%)$ & $10(16.7 \%)$ & $P<0.01$ \\
Lymph node enlargement & $10(62.5 \%)$ & $53(88.3 \%)$ & $P<0.01$ \\
\hline
\end{tabular}

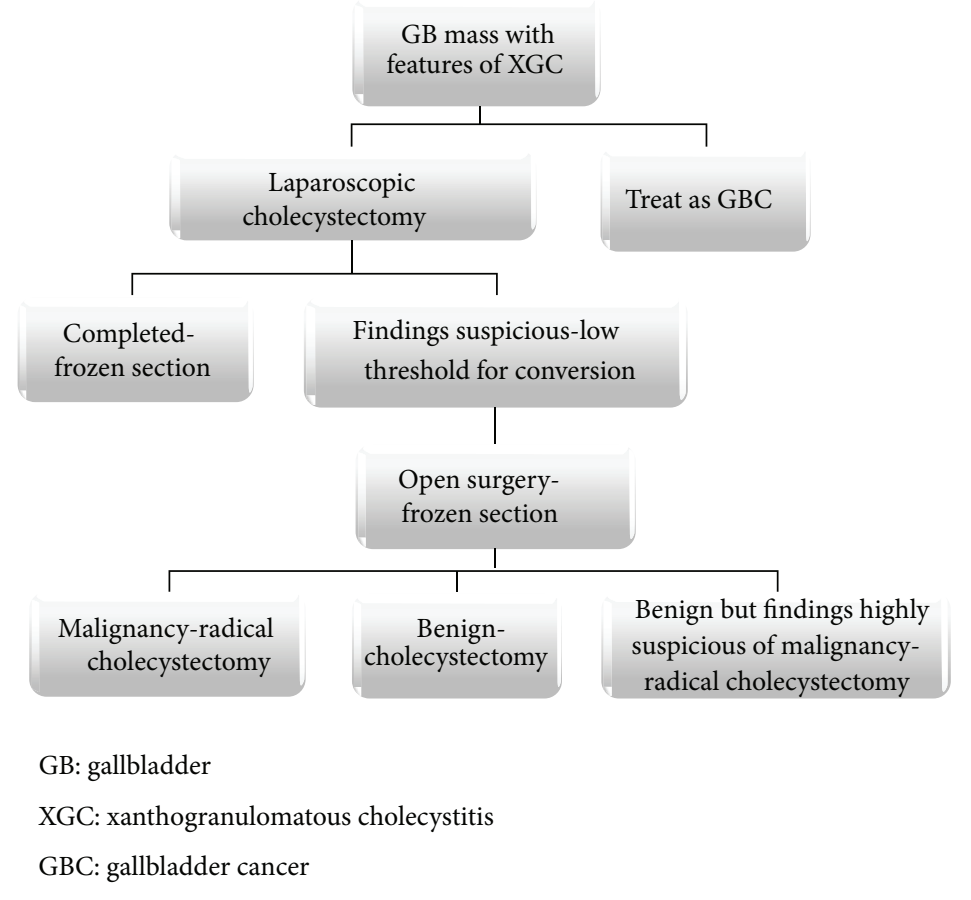

FIGURE 4: Algorithm for management of gallbladder mass with features of XGC.

\section{Discussion}

XGC can exhibit similar imaging and intraoperative findings as those of GBC and are easily misdiagnosed, often leading to unnecessary radical surgery $[1,4,13]$. An increased awareness combined with an increased accuracy of preoperative and intraoperative diagnosis and an algorithmic approach to XGC could help avoid extended resections. Analogous to the results of a Chinese study, our data suggests that the presence of abdominal pain, acute cholecystitis, choledocholithiasis, and cholelithiasis may portend a diagnosis of XGC [6]. The role of tumour markers remains unresolved, with some studies showing no significant correlations with diagnosis $[2,3,5,6]$. In our study, raised levels of tumour markers correlated well with the incidence of GBC and could be used in the differentiation of XGC from GBC.

Extravasation of bile into the gallbladder wall with involvement of Rokitansky-Aschoff sinuses is a potential precipitating factor for XGC [6-12]. These lead to formation of submucosal abscesses or xanthogranulomas, which show up on CECT as submucosal hypoattenuated nodules occupying large areas of the gallbladder wall, a sign highly suggestive of XGC. Other features like the continuous mucosal line in a thickened gallbladder wall and the presence of gallstones in a background of chronic gallbladder disease have been reported as being highly suggestive of XGC [6-12]. The radiological findings indicative of XGC in our series concurred with those reported in literature.

EUS-guided FNA (EUS-FNA) is a useful modality for sampling various targets. Even though EUS-FNA is a feasible and safe method for obtaining samples, its role in the diagnostic workup of gallbladder lesions remains undefined. While a positive FNAC confirms the diagnosis of GBC, a negative sample does not shed much light. The overall sampling adequacy is reported to be $86 \%$. The accuracy of EUSFNA for detecting malignancy and for the final diagnosis is approximately $93 \%$ and $80 \%$, respectively $[13,14]$. Sampling errors in the form of samples from nonrepresentative areas along with a confounding factor of coexistence of XGC and GBC limit the widespread applicability of EUS-FNA in $\mathrm{XGC}[13,14]$. Intraoperative frozen section examination is an efficient method for exclusion of GBC. Its liberal use also helps rule out the simultaneous occurrence of GBC/XGC, thereby guiding optimum surgery [2, 6, 15-17]. Frozen section 
in combination with immunohistochemistry has shown to be highly sensitive in their ability to differentiate XGC from GBC [18].

Controversy exists regarding the use of laparoscopic cholecystectomy (LC) in patients with XGC [19-21]. The intense chronic inflammatory process can make the procedure arduous and hazardous, and hence in any patient with a difficult laparoscopic cholecystectomy, an on-table differential diagnosis of XGC must be entertained amongst others. Multiple series have attested to the safety of LC in $\mathrm{XGC}$, with no increase in the morbidity as compared to an open procedure. There is indeed a higher incidence of conversion to an open procedure, but this low threshold for conversion to open surgery enables a better assessment of the lesion and results in superior outcomes with regard to mortality and morbidity [1, 3, 19-21].

A combination of clinical, radiological factors combined with a liberal application of intraoperative frozen section examination can aid in the diagnosis and surgery for XGC. To help guide the surgeon towards a structured and rationalized management of XGC, based on our study, a simple algorithm has been proposed (Figure 4). As suggested in our algorithm, despite all radiological and frozen section analyses, a high index of suspicion of GBC on the part of the operating surgeon warrants a radical surgery.

\section{Conclusion}

Differentiating XGC from GBC is a diagnostic conundrum. Making this distinction preoperatively or intraoperatively is difficult, and a definitive diagnosis still necessitates a histopathological examination. An accurate preoperative diagnosis requires an integrated review of clinical and characteristic radiological features, the presence of which may help avoid radical resection and avoidable morbidity in selected cases.

\section{Conflict of Interests}

The authors declare that there is no conflict of interests regarding the publication of this paper.

\section{Authors' Contribution}

Dr. Ashwin Rammohan, Dr. Sathya D. Cherukuri, and Professor Jeswanth Sathyanesan contributed to conception and design, acquisition, analysis, and interpretation of data. Dr. Ashwin Rammohan, Dr. Sathya D. Cherukuri, Professor Jeswanth Sathyanesan, and Professor Ravichandran Palaniappan drafted the paper and revised it critically for important intellectual content. Professor Jeswanth Sathyanesan, Professor Ravichandran Palaniappan, and Professor Manoharan Govindan gave the final approval of the version to be published.

\section{References}

[1] V. G. Guzman, "Xanthogranulomatous cholecystitis: 15 years' experience," World Journal of Surgery, vol. 28, no. 3, pp. 254$257,2004$.
[2] T. Yang, B.-H. Zhang, J. Zhang, Y.-J. Zhang, X.-Q. Jiang, and M.-C. Wu, "Surgical treatment of xanthogranulomatous cholecystitis: experience in 33 cases," Hepatobiliary \& Pancreatic Diseases International, vol. 6, no. 5, pp. 504-508, 2007.

[3] T. Yang, B.-H. Zhang, J. Zhang, Y.-J. Zhang, X.-Q. Jiang, and M.-C. Wu, "Surgical treatment of xanthogranulomatous cholecystitis: experience in 33 cases," Hepatobiliary and Pancreatic Diseases International, vol. 6, no. 5, pp. 504-508, 2007.

[4] A. Spinelli, G. Schumacher, A. Pascher et al., "Extended surgical resection for xanthogranulomatous cholecystitis mimicking advanced gallbladder carcinoma: a case report and review of literature," World Journal of Gastroenterology, vol. 12, no. 14, pp. 2293-2296, 2006.

[5] D. Sharma, R. Babu, G. Sood, G. Kapoor, R. S. Solanki, and S. Thomas, "Xanthogranulomatous cholecystitis masquerading as malignancy with liver metastasis," ANZ Journal of Surgery, vol. 79, no. 12, pp. 946-947, 2009.

[6] L.-F. Zhang, C.-S. Hou, J.-Y. Liu et al., "Strategies for diagnosis of xanthogranulomatous cholecystitis masquerading as gallbladder cancer," Chinese Medical Journal, vol. 125, no. 1, pp. 109-113, 2012.

[7] K. A. Chun, H. K. Ha, E. S. Yu et al., "Xanthogranulomatous cholecystitis: CT features with emphasis on differentiation from gallbladder carcinoma," Radiology, vol. 203, no. 1, pp. 93-97, 1997.

[8] P. N. Kim, S. H. Lee, G.-Y. Gong et al., "Xanthogranulomatous cholecystitis: radiologic findings with histologic correlation that focuses on intramural nodules," American Journal of Roentgenology, vol. 172, no. 4, pp. 949-953, 1999.

[9] K. Uchiyama, S. Ozawa, M. Ueno et al., "Xanthogranulomatous cholecystitis: the use of preoperative CT findings to differentiate it from gallbladder carcinoma," Journal of Hepato-BiliaryPancreatic Surgery, vol. 16, no. 3, pp. 333-338, 2009.

[10] J. A. Parra, O. Acinas, J. Bueno, A. Güezmes, M. A. Fernández, and M. C. Fariñas, "Xanthogranulomatous cholecystitis: clinical, sonographic, and CT findings in 26 patients," American Journal of Roentgenology, vol. 174, no. 4, pp. 979-983, 2000.

[11] R. Shuto, H. Kiyosue, E. Komatsu et al., "CT and MR imaging findings of xanthogranulomatous cholecystitis: correlation with pathologic findings," European Radiology, vol. 14, no. 3, pp. 440446, 2004.

[12] S. Goshima, S. Chang, J. H. Wang, M. Kanematsu, K. T. Bae, and M. P. Federle, "Xanthogranulomatous cholecystitis: diagnostic performance of CT to differentiate from gallbladder cancer," European Journal of Radiology, vol. 74, no. 3, pp. e79-e83, 2010.

[13] G. N. Srinivas, S. Sinha, N. Ryley, and P. W. Houghto, "Perfidious gallbladders-a diagnostic dilemma with xanthogranulomatous cholecystitis," Annals of The Royal College of Surgeons of England, vol. 89, pp. 168-172, 2007.

[14] S. Hijioka, M. A. Mekky, V. Bhatia et al., "Can EUS-guided FNA distinguish between gallbladder cancer and xanthogranulomatous cholecystitis?" Gastrointestinal Endoscopy, vol. 72, no. 3, pp. 622-627, 2010.

[15] A. H. Kwon, Y. Matsui, and Y. Uemura, "Surgical procedures and histopathologic findings for patients with xanthogranulomatous cholecystitis," Journal of the American College of Surgeons, vol. 199, no. 2, pp. 204-210, 2004.

[16] J. Pinocy, A. Lange, C. König, E. Kaiserling, H. D. Becker, and S. M. Kröber, "Xanthogranulomatous cholecystitis resembling carcinoma with extensive tumorous infiltration of the liver and colon," Langenbeck's Archives of Surgery, vol. 388, no. 1, pp. 4851, 2003. 
[17] R. P. Krishna, A. Kumar, R. K. Singh, S. Sikora, R. Saxena, and V. K. Kapoor, "Xanthogranulomatous inflammation stricture of extrahepatic biliary tract: presentation and surgical management," Journal of Gastrointestinal Surgery, vol. 12, no. 5, pp. 836841, 2008.

[18] R. V. R. Rao, A. Kumar, S. S. Sikora, R. Saxena, and V. K. Kapoor, "Xanthogranulomatous cholecystitis: differentiation from associated gall bladder carcinoma," Tropical Gastroenterology, vol. 26, no. 1, pp. 31-33, 2005.

[19] A. Alvi, I. Jalbani, G. Murtaza, and A. Hameed, "Outcomes of Xanthogranulomatous cholecystitis in laparoscopic era: a retrospective Cohort study," Journal of Minimal Access Surgery, vol. 9, no. 3, pp. 109-115, 2013.

[20] G. Guzmán-Valdivia, "Xanthogranulomatous cholecystitis in laparoscopic surgery," Journal of Gastrointestinal Surgery, vol. 9, no. 4, pp. 494-497, 2005.

[21] G. Srikanth, A. Kumar, R. Khare et al., "Should laparoscopic cholecystectomy be performed in patients with thick-walled gallbladder?" Journal of Hepato-Biliary-Pancreatic Surgery, vol. 11, pp. 40-44, 2004. 


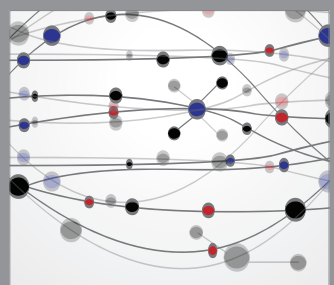

The Scientific World Journal
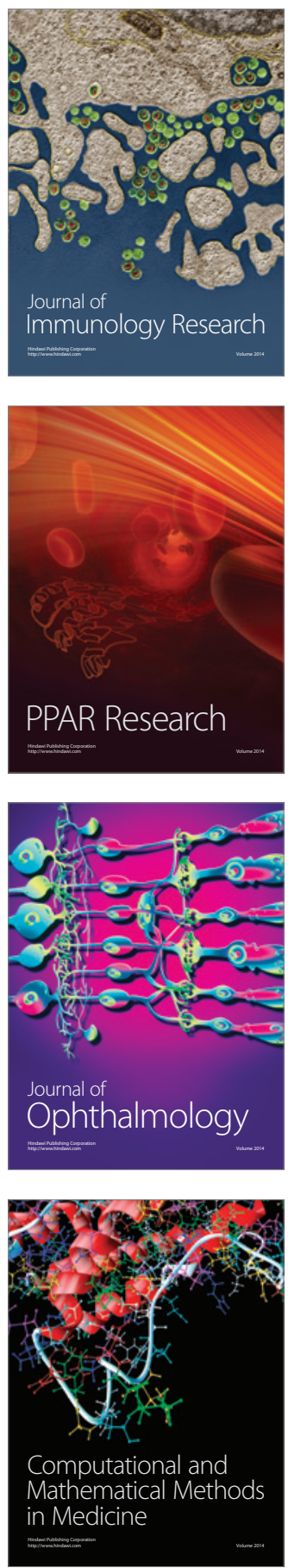

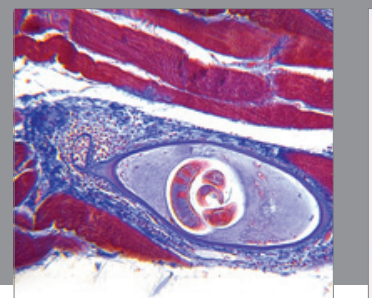

Gastroenterology

Research and Practice
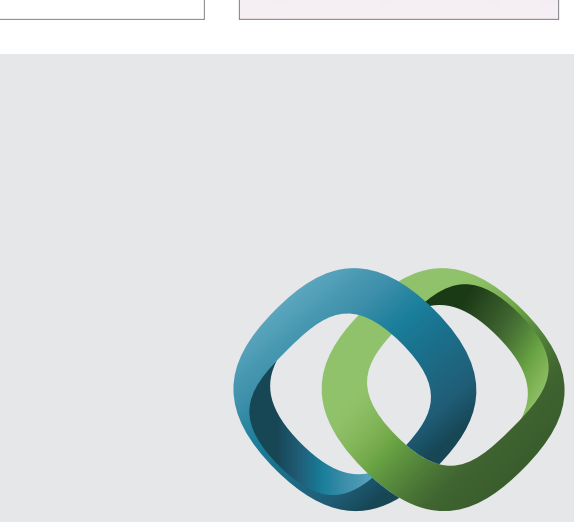

\section{Hindawi}

Submit your manuscripts at

http://www.hindawi.com
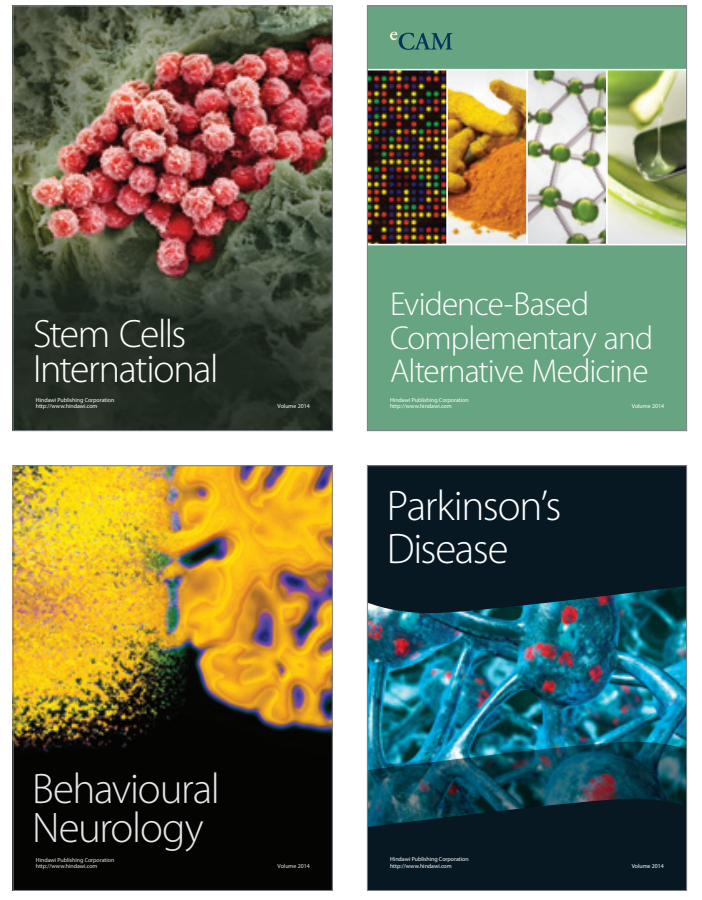
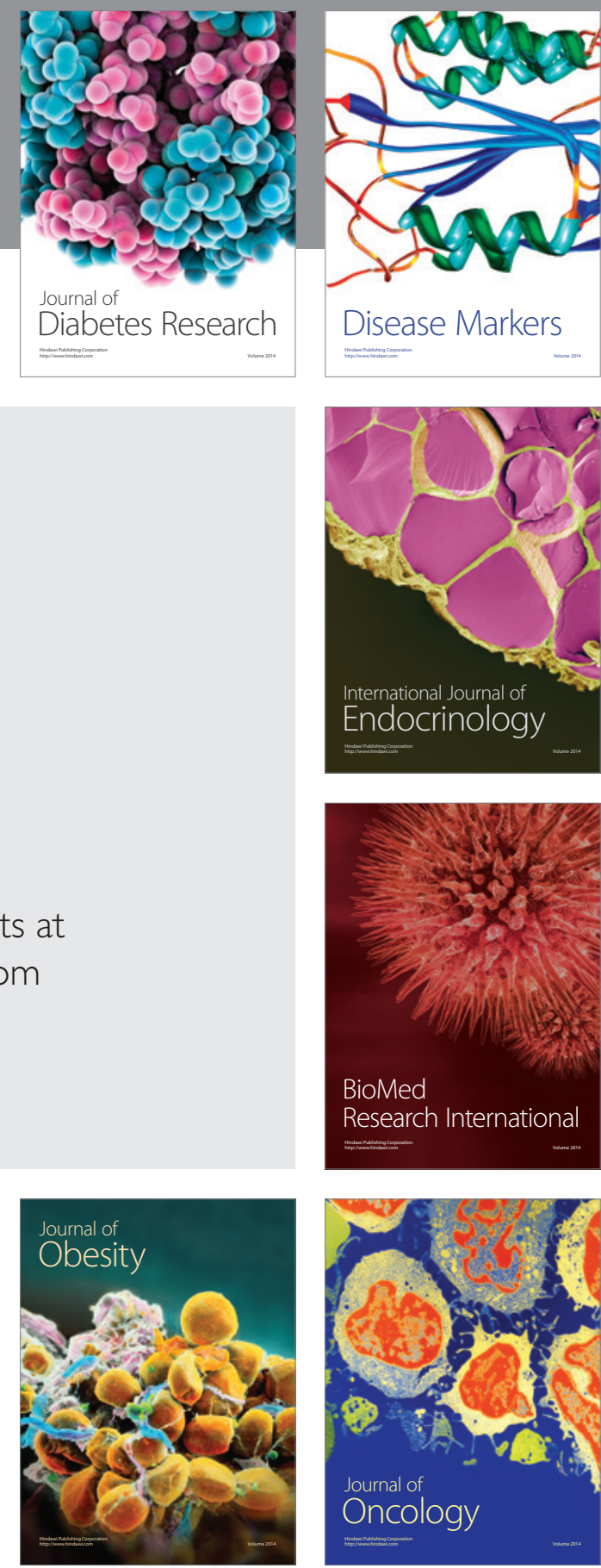

Disease Markers
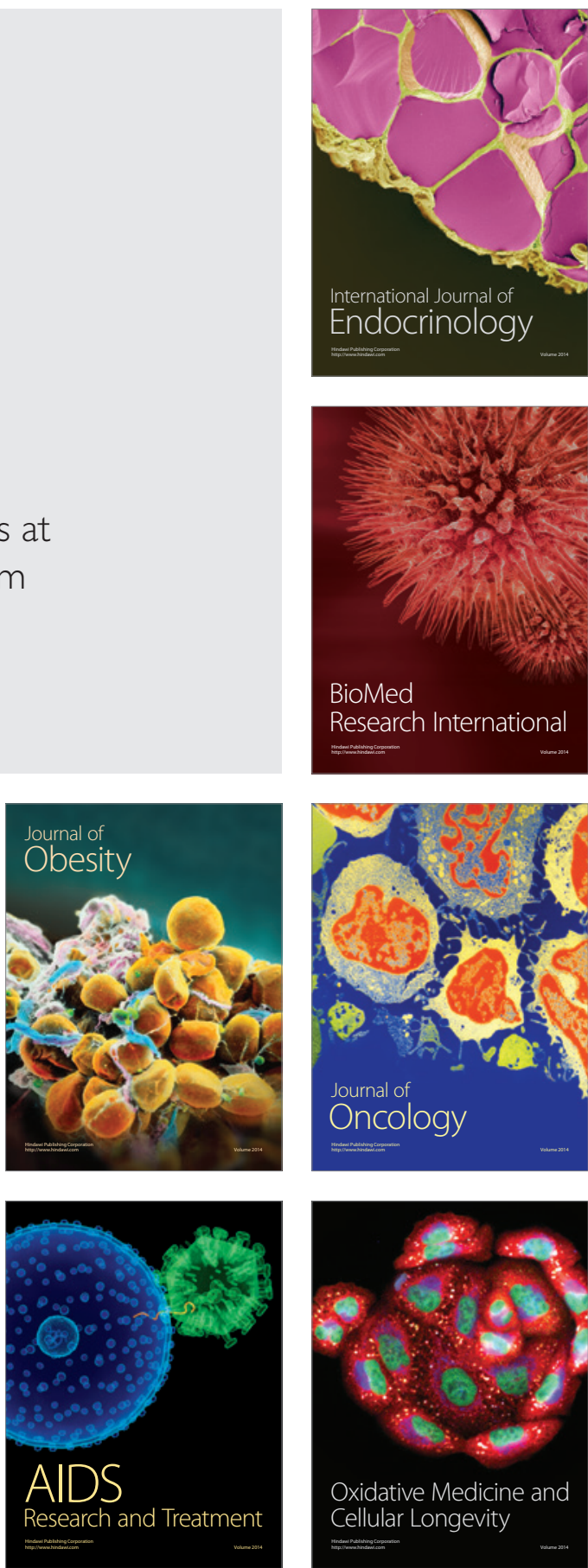